\title{
Wavelength tunable MEMS VCSELs for OCT imaging
}

Sahoo, Hitesh Kumar; Ansbæk, Thor; Ottaviano, Luisa; Semenova, Elizaveta; Hansen, Ole; Yvind, Kresten

\section{Published in:}

Proceedings of SPIE

Link to article, DOI:

$10.1117 / 12.2289545$

Publication date:

2018

Document Version

Publisher's PDF, also known as Version of record

Link back to DTU Orbit

Citation (APA):

Sahoo, H. K., Ansbæk, T., Ottaviano, L., Semenova, E., Hansen, O., \& Yvind, K. (2018). Wavelength tunable MEMS VCSELLs for OCT imaging. In Proceedings of SPIE (Vol. 10552). [105520I ] SPIE - International Society for Optical Engineering. Proceedings of SPIE - The International Society for Optical Engineering https://doi.org/10.1117/12.2289545

\section{General rights}

Copyright and moral rights for the publications made accessible in the public portal are retained by the authors and/or other copyright owners and it is a condition of accessing publications that users recognise and abide by the legal requirements associated with these rights.

- Users may download and print one copy of any publication from the public portal for the purpose of private study or research.

- You may not further distribute the material or use it for any profit-making activity or commercial gain

- You may freely distribute the URL identifying the publication in the public portal 


\section{Wavelength tunable MEMS VCSELs for OCT imaging}

Hitesh Kumar Sahoo, Thor Ansbæk, Luisa Ottaviano, Elizaveta Semenova, Ole Hansen, et al.

Hitesh Kumar Sahoo, Thor Ansbæk, Luisa Ottaviano, Elizaveta Semenova, Ole Hansen, Kresten Yvind, "Wavelength tunable MEMS VCSELs for OCT imaging," Proc. SPIE 10552, Vertical-Cavity Surface-Emitting Lasers XXII, 1055201 (19 February 2018); doi: 10.1117/12.2289545

SPIE. Event: SPIE OPTO, 2018, San Francisco, California, United States 


\title{
Wavelength tunable MEMS VCSELs for OCT imaging
}

\author{
Hitesh Kumar Sahoo ${ }^{\mathrm{a}}$, Thor Ansbæk ${ }^{\mathrm{b}}$, Luisa Ottaviano ${ }^{\mathrm{a}}$, Elizaveta Semenova ${ }^{\mathrm{a}}$, Ole Hansen ${ }^{\mathrm{c}}$, \\ and Kresten Yvind ${ }^{\mathrm{a}}$ \\ ${ }^{a}$ DTU Fotonik, Building 343, Ørsteds Plads, DK-2800 Kongens Lyngby, Denmark \\ ${ }^{\mathrm{b}}$ OCTLight Aps, Scion DTU, DK-2800 Kongens Lyngby, Denmark \\ ${ }^{\mathrm{c} D T U}$ Nanotech, Building 345C, Ørsteds Plads, DK-2800 Kongens Lyngby, Denmark
}

\begin{abstract}
MEMS VCSELs are one of the most promising swept source (SS) lasers for optical coherence tomography (OCT) and one of the best candidates for future integration with endoscopes, surgical probes and achieving an integrated OCT system. However, the current MEMS-based SS are processed on the III-V wafers, which are small, expensive and challenging to work with. Furthermore, the actuating part, i.e., the MEMS, is on the top of the structure which causes a strong dependence on packaging to decrease its sensitivity to the operating environment. This work addresses these design drawbacks and proposes a novel design framework. The proposed device uses a high contrast grating mirror on a Si MEMS stage as the bottom mirror, all of which is defined in an SOI wafer. The SOI wafer is then bonded to an InP III-V wafer with the desired active layers, thereby sealing the MEMS. Finally, the top mirror, a dielectric DBR (7 pairs of $\left.\mathrm{TiO}_{2}-\mathrm{SiO}_{2}\right)$, is deposited on top. The new device is based on a silicon substrate with MEMS defined on a silicon membrane in an enclosed cavity. Thus the device is much more robust than the existing MEMS VCSELs. This design also enables either a two-way actuation on the MEMS or a smaller optical cavity (pull-away design), i.e., wider FSR (Free Spectral Range) to increase the wavelength sweep. Fabrication of the proposed device is outlined and the results of device characterization are reported.
\end{abstract}

Keywords: MEMS, VCSEL, OCT, wavelength tunable, low threshold

\section{INTRODUCTION}

Swept source (SS) - optical coherence tomography (OCT) technologies are already established as superior to spectral domain (SD) OCT and time domain (TD) OCT. They are much faster ${ }^{1}$ and with a better signal-tonoise-ratio (SNR). ${ }^{2}$ Micro-electro-mechanical system (MEMS) vertical cavity surface emitting lasers (VCSELSs), in particular are promising candidates for the light source of the future OCT technologies. They are more future proof since they are wafer based technologies and have the potential to deliver better performance than the other competing SS light source technologies. However, Drexler et al. ${ }^{3}$ point out, that the perfect SS does not exist. The existing MEMS VCSEL designs ${ }^{4-7}$ are based on the III-V wafers, which are small, expensive and challenging to work with. In addition, the actuating part (MEMS) is on the top and is open to the working environment until packaged. Ideally, the MEMS/actuating part should be in a sealed cavity in order to have a good control of the mechanical dynamics. Otherwise, any change in temperature or pressure may result in water condensation on the actuating electrodes which may lead to stiction, that is destructive to the device. This work addresses these drawbacks and proposes a novel MEMS VCSEL design. The proposed design deviates from the usual MEMS VCSEL design framework and makes a step closer towards a more robust device.

\section{DESIGN}

There are a number of existing MEMS VCSEL designs, ${ }^{5-9}$ they are, however, all based on the same design framework. They borrow the structure from a VCSEL design which is heavily dependent on III-V growth, which is quite challenging. From a design perspective VCSELs are multilayered structures without moving parts. Unlike VCSELs, MEMS VCSELs have a characteristic built-in air-gap which is tuned using a movable mirror. In

Further author information: (Send correspondence to H.K.S.)

H.K.S.: E-mail: hikus@fotonik.dtu.dk

Vertical-Cavity Surface-Emitting Lasers XXII, edited by Chun Lei, Kent D. Choquette, Proc. of SPIE

Vol. 10552, 1055201 ? (c) 2018 SPIE · CCC code: 0277-786X/18/\$18 · doi: 10.1117/12.2289545 
addition, the III-V material is used to define the MEMS element, which is not ideal from a mechanical robustness point of view. Furthermore, the air-gap is a part of the optical cavity and contributes to the MEMS dynamics. Thus, the air-gap needs to be a controlled environment. There is also the risk of damaging the MEMS element due to water condensation. These fundamental issues are addressed here and a new design concept is proposed.

Figure 1 shows the design evolution of the existing MEMS VCSEL designs from VCSELs. The bottom mirror is in all cases a grown low index contrast DBR mirror on top of a III-V substrate. The gain material is either grown along with the bottom mirror ${ }^{4}$ or bonded to the bottom mirror. ${ }^{5}$ Since the bottom mirror is thick and is fixed on top of the substrate, it is mostly the top mirror, which is actuated. The top mirror is in some cases modified to be a dielectric distributed Bragg reflector (DBR) stack mirror ${ }^{5}$ or a high contrast grating (HCG) mirror. ${ }^{4,7}$ Both approaches are huge improvements in terms of the resulting mirror thickness, with the latter as the best. The challenge here is to achieve a very high reflectivity on the order of $99.9 \%$. The dielectric DBR does a good job owing to the higher contrast difference than that of the III-V counterpart. The HCG has a very high reflectivity owing to the near wavelength thickness with the high contrast grating structure. Nevertheless, the actuating part is still the top mirror based on dielectric or III-V materials.

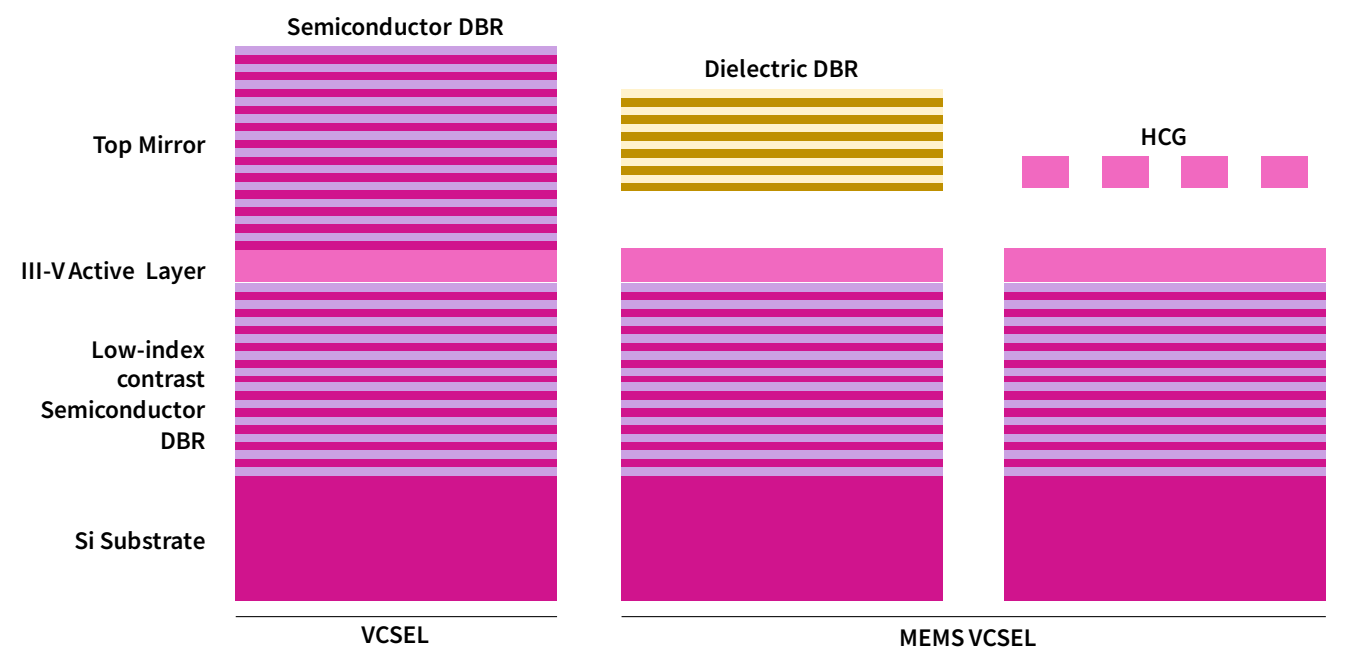

Figure 1. Schematic illustration comparing the design framework of VCSELs and existing MEMS VCSELs.

Thus the proposed design addresses two main issues: the heavy dependence on III-V materials and the open actuation element. The best processing platform choice is the more established Si platform. There is a lot of established knowledge on Si processing and the Si substrate is cheaper and mechanically more robust to work with. With a Si substrate, the options for the bottom mirror are a dielectric DBR or a HCG. Defining a HCG on the device Si layer of a SOI substrate and actuating it, addresses the second issue too. A HCG is thus the perfect choice for the bottom mirror. The active material still remains and the top mirror can be defined by a dielectric DBR. A sketch of the re-designed MEMS VCSEL is shown in Figure 2. To summarize, the proposed device is a Si substrate based MEMS VCSEL defined with a HCG mirror as the bottom mirror and a dielectric DBR as the top mirror, and the actuating part is defined on Si and resides in a sealed cavity.

The new design offers many advantages compared to the traditional approach. First and foremost, it is a huge step towards the more established and standard silicon platform and it conviniently includes broadband dielectric DBRs. Encapsulating the actuating part (HCG on a MEMS frame) is another major improvement. It enables the designer to have good control of the operating atmosphere of the MEMS - vacuum or nitrogen or another gas which contributes the desired damping. Furthermore, the actuating part is now defined on Si, which is a much better material than its III-V counterparts in terms of robustness and reliability.

The design also brings a modular approach to the design of MEMS VCSELs, the possibility to sweep in 2 directions and it results in a smaller cavity, without being limited by MEMS pull-in constraints. Unlike the 
traditional approach, all the elements can be designed and fabricated independently. As a result, the design can be modified quite fast to accommodate any change in emission wavelength based on the application area. Another interesting aspect of the device is its ability to tune in two directions. The MEMS can be actuated by applying a voltage between the device Si-layer of the silicon on insulator (SOI) and either the Si substrate or the InP layer on the top. This is particularly important to increase the tuning range, which is limited to $1 / 3 \mathrm{rd}$ of the distance between the electrodes for electrostatic actuation. Here, the tuning range is extended to $1 / 3$ of the total air gap (top and bottom of the MEMS). In addition, to achieve a smaller cavity, the top air-gap can be reduced while only the bottom air-gap is tuned. In other words, the proposed design enables a pull-away actuation which is impossible in the existing devices.

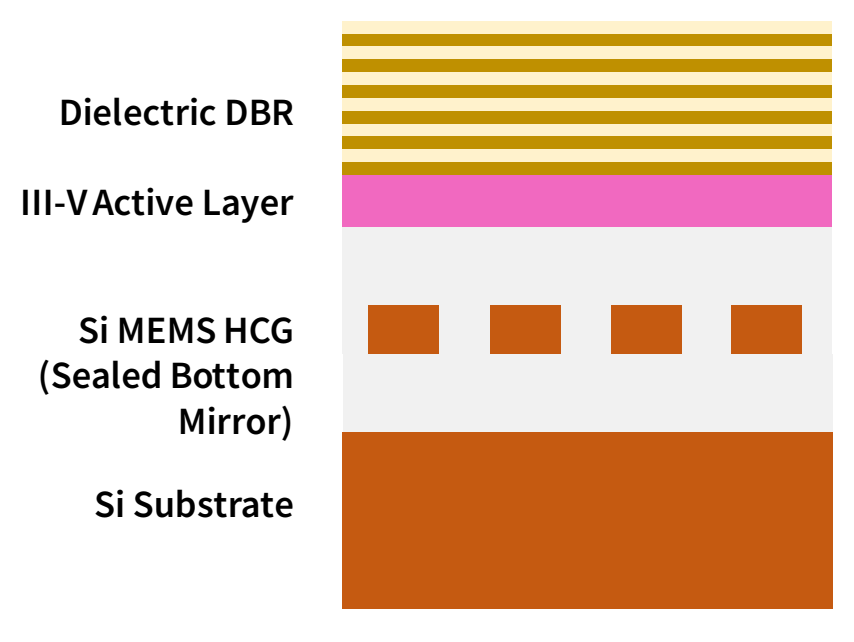

Figure 2. Sketch of the proposed MEMS VCSEL.

\section{FABRICATION}

One of the key features of this device design is the flexibility it brings into the fabrication process. The bottom mirror, a MEMS HCG, is first defined in the device layer of an SOI wafer while the active material - quantum wells (QWs) are grown on an $\mathrm{InP}$ substrate independently. Then, the two wafers are bonded together, the InP substrate removed and finally, $\mathrm{SiO}_{2} / \mathrm{TiO}_{2} \mathrm{DBR}$ layers are deposited. Thus, all the critical processing steps with strict tolerances (HCG patterning with E-beam, growth of QWs in MOVPE and wafer bonding) are processed independently. Only then, the processes with less strict tolerances (substrate removal, contact etching) follow. Direct-bonding using ultra-thin $\mathrm{Al}_{2} \mathrm{O}_{3}$ at the bonding interfaces followed by an anneal at $300{ }^{\circ} \mathrm{C}$ was chosen to bond the SOI with the InP wafer. ${ }^{10}$ This procedure helps to achieve a good bond (good enough to survive substrate removal and all the post processing) and avoid any adhesives or unwanted layers in the optical path.

\section{CHARACTERIZATION}

A simple microscope-based optical setup was used for characterizing the fabricated MEMS VCSEL. A commercially available $980 \mathrm{~nm}$ laser diode was used as the pump source. A near-infrared (NIR) InGaAs camera mounted on top of the microscope was used for looking at the sample and position the pump beam. The laser output was collected on an optical spectrum analyzer (OSA), which was connected to a GPIB bus for data collection and analysis on a computer. Figure 3 shows a schematic of the optical setup for the MEMS VCSEL characterization. In this setup, the pump laser and the MEMS VCSEL laser output share most of the optical path. The light from the source was fed through an optical fiber into a microscope with the help of a piezo stage and a collimating lens. The piezo stage helped in positioning and alignment of the pump beam. The pump beam was then directed at the sample using a dichroic mirror at 45 degrees and a focusing objective lens (either 20X or 50X). The sample 
was placed on a 2D translation stage, which had a vacuum holder and was temperature controlled. The laser output from the fabricated device was collected back the same way, through the objective lens, dichroic mirror and into the optical fiber. A WDM coupler was used between the pump laser and piezo stage to filter out the $1550 \mathrm{~nm}$ component (MEMS VCSEL laser output). It was then connected to an OSA. A part of the output was also used as a feedback for optimal positioning of the fiber at the back of the microscope. Inside the microscope, a small fraction of the MEMS VCSEL output was transmitted through the dichroic mirror. It was further filtered using a long-pass $1310 \mathrm{~nm}$ filter to remove the pump and just look at the MEMS VCSEL output using the NIR camera. It was helpful in quick scanning through the fabricated chip and for manual positioning of the pump beam and probes.

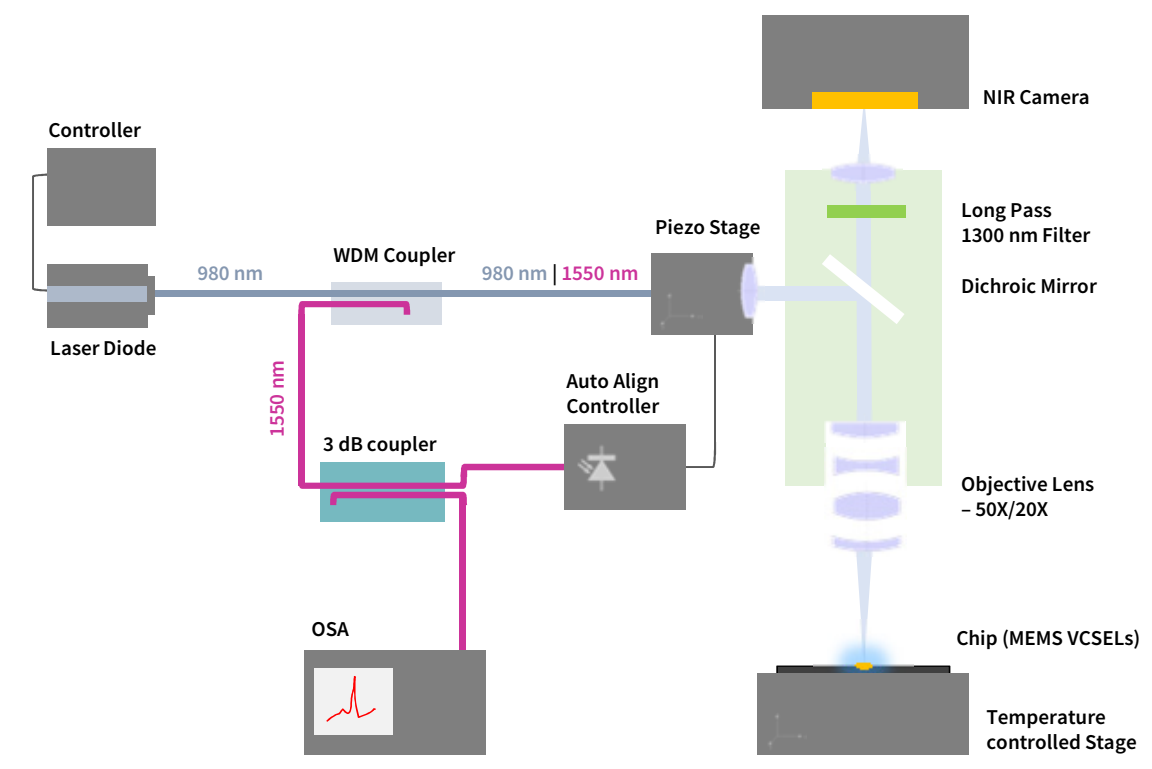

Figure 3. Illustration of the characterization setup using a $980 \mathrm{~nm}$ laser for pumping. A microscope based setup was used for the pumping and collection of lasing light. A NIR camera was added on the top of the microscope to look and position the sample.

\section{RESULTS}

The threshold for lasing of the fabricated MEMS VCSEL was obtained by plotting the peak lasing power of the spectrum at different pump laser diode current values (Figure 4). The threshold input power (delivered on the chip) at room temperature for the laser was approximately $5.5 \mathrm{~mW}$, which translates to around $1.4 \mathrm{~mW}$ of absorbed power (based on an assumption of $25 \%$ absorption for $980 \mathrm{~nm}$ pumping). This is equivalent to an electrical injection current of $1 \mathrm{~mA}$, which is comparable to some well-designed VCSELs. ${ }^{4,11-14}$ The maximum power output achieved for the device is approx. $0.1 \mathrm{~mW}$ (after correction for $12 \mathrm{~dB}$ loss in the optical path). There are some minor deviations on the threshold graph from the standard curve. This was because of an unintended change in focus of the microscope stage (due to the weight of the NIR camera).

The fabricated MEMS VCSEL, unlike existing MEMS VCSELs, uses three electrodes for actuation. The top electrode is defined by an n-doped InP layer. The Si MEMS layer forms the centre electrode and the $\mathrm{Si}$ substrate constitutes the bottom electrode. Application of a voltage between the center electrode and either top or bottom electrode creates an electrostatic attractive force between the two electrodes. This moves the mirror and changes the cavity length. As a result, we observe a change in the lasing wavelength. Figure 5 shows the change in lasing wavelength at different applied voltages. Application of a voltage between the Si MEMS and 


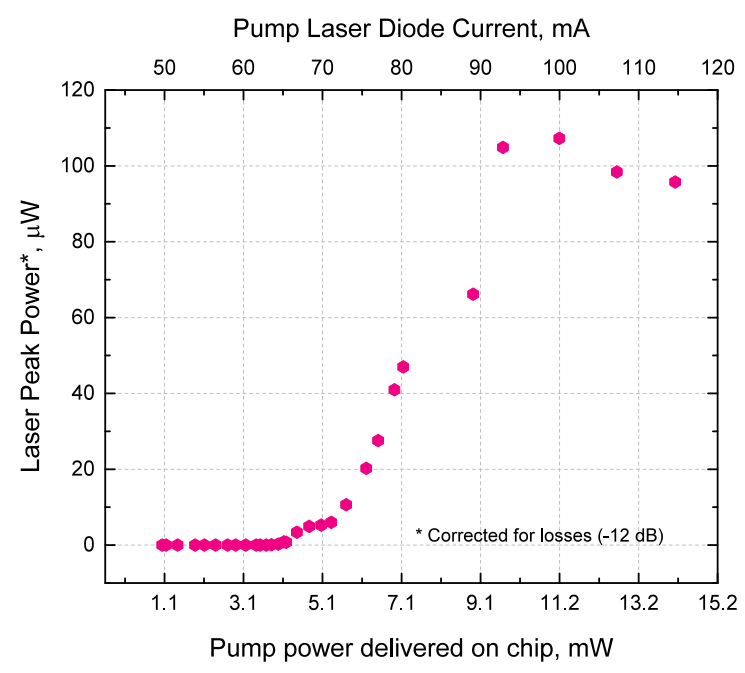

Figure 4. Laser power (max in optical spectra) plotted against the pump power. The collected laser power is corrected for the losses in the measurement system.

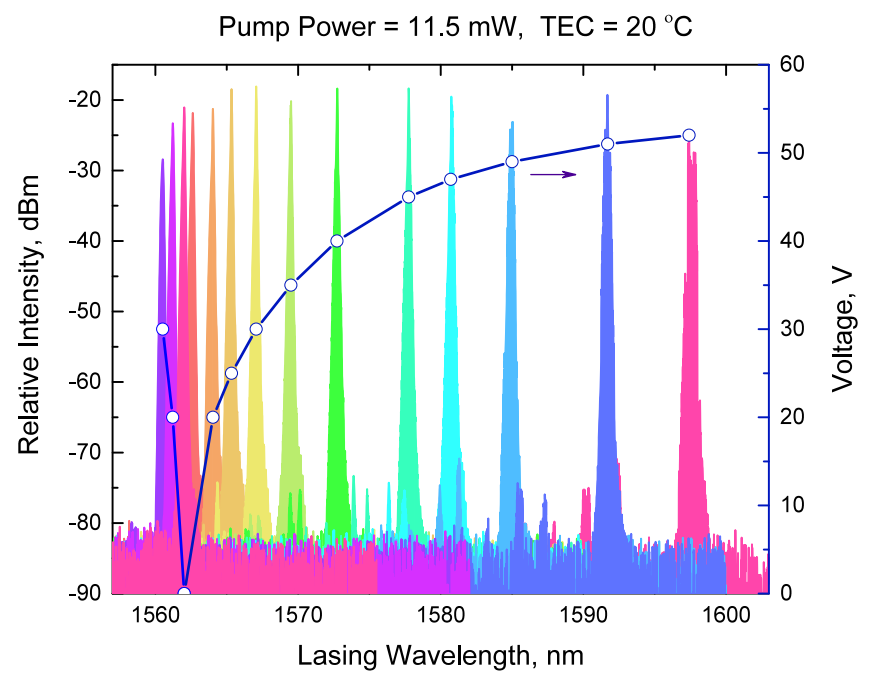

Figure 5. Lasing spectra showing wavelength tuning achieved at different actuation voltages.

Si substrate increases the cavity length of VCSEL and we see laser tuning towards higher wavelength. A tuning range of around $40 \mathrm{~nm}$ was achieved. Similarly, a voltage between the Si MEMS and InP reduced the cavity length which moved the lasing curve to lower wavelength. Unfortunately, a wider tuning to lower wavelengths was not attained. The losses increased quite fast which restricted the tuning to just a couple of nm. Possible explanations include improper (not optimized) contacts, strong heating redshifting the gain spectrum or simply a limitation from the gain bandwidth. More investigations are required to trace the problem. Nevertheless, a total of $40 \mathrm{~nm}$ of DC tuning was achieved on the first generation fabricated MEMS VCSELs.

\section{CONCLUSION}

We have discussed a new MEMS VCSEL design, fabricated and characterized the new design. The new design represents a major step away from an all III-V material device towards a design based on a rugged Si platform. Characterization of the fabricated device show lasing with a low threshold at $1.4 \mathrm{~mW}$, which is equivalent to 1 
$\mathrm{mA}$ of electrical pumping. A wide DC tuning range of $40 \mathrm{~nm}$ was achieved with some degree of bi-directional tuning, which should be improved by future optimization. The new design has the potential to become the perfect SS of the future OCT systems, in particular since the sensitive movable part resides in a sealed cavity.

\section{ACKNOWLEDGMENTS}

The authors would like to acknowledge the financial support from the Danish Innovation Foundation through the HERON project, the Villum Center of Excellence NATEC II, and the National Basic Research Foundation center SPOC (No. DNRF123).

\section{REFERENCES}

[1] Wieser, W., Biedermann, B. R., Klein, T., Eigenwillig, C. M., and Huber, R., "Multi-megahertz oct: High quality 3d imaging at 20 million a-scans and 4.5 gvoxels per second," Optics express 18(14), 14685-14704 (2010).

[2] Grulkowski, I., Liu, J. J., Potsaid, B., Jayaraman, V., Lu, C. D., Jiang, J., Cable, A. E., Duker, J. S., and Fujimoto, J. G., "Retinal, anterior segment and full eye imaging using ultrahigh speed swept source oct with vertical-cavity surface emitting lasers," Biomedical optics express 3(11), 2733-2751 (2012).

[3] Drexler, W., Liu, M., Kumar, A., Kamali, T., Unterhuber, A., and Leitgeb, R. A., "Optical coherence tomography today: speed, contrast, and multimodality," Journal of biomedical optics 19(7), 071412-071412 (2014).

[4] Ansbæk, T., Chung, I.-S., Semenova, E. S., and Yvind, K., "1060-nm tunable monolithic high index contrast subwavelength grating vcsel," IEEE Photonics Technology Letters 25(4), 365-367 (2013).

[5] Jayaraman, V., Cole, G., Robertson, M., Burgner, C., John, D., Uddin, A., and Cable, A., "Rapidly swept, ultra-widely-tunable $1060 \mathrm{~nm}$ mems-vcsels," Electronics letters 48(21), 1331-1333 (2012).

[6] Gierl, C., Gruendl, T., Debernardi, P., Zogal, K., Grasse, C., Davani, H., Böhm, G., Jatta, S., Küppers, F., Meißner, P., et al., "Surface micromachined tunable $1.55 \mu \mathrm{m}$-vcsel with $102 \mathrm{~nm}$ continuous single-mode tuning," Optics Express 19(18), 17336-17343 (2011).

[7] Chase, C., Rao, Y., Hofmann, W., and Chang-Hasnain, C. J., "1550 nm high contrast grating vcsel," Optics express 18(15), 15461-15466 (2010).

[8] Riemenschneider, F., Maute, M., Halbritter, H., Boehm, G., Amann, M.-C., and Meissner, P., "Continuously tunable long-wavelength mems-vcsel with over 40-nm tuning range," IEEE Photonics Technology Letters 16(10), 2212-2214 (2004).

[9] Ansbæk, T., Chung, I.-S., Semenova, E. S., Hansen, O., and Yvind, K., "Resonant mems tunable vcsel," IEEE Journal of Selected Topics in Quantum Electronics 19(4), 1702306-1702306 (2013).

[10] Sahoo, H. K., Ottaviano, L., Zheng, Y., Hansen, O., and Yvind, K., "Low temperature bonding of heterogeneous materials using al2o3 as an intermediate layer," Journal of Vacuum Science 85 Technology B, Nanotechnology and Microelectronics: Materials, Processing, Measurement, and Phenomena 36(1), 011202 (2017).

[11] Ortsiefer, M., Shau, R., Böhm, G., Köhler, F., and Amann, M.-C., "Low-threshold index-guided $1.5 \mu \mathrm{m}$ long-wavelength vertical-cavity surface-emitting laser with high efficiency," Applied Physics Letters 76(16), 2179-2181 (2000).

[12] Ortsiefer, M., Furfanger, M., Rosskopf, J., Bohm, G., Kohler, F., Lauer, C., Maute, M., Hofmann, W., and Amann, M.-C., "Singlemode 1.55/spl mu/m vcsels with low threshold and high output power," Electronics Letters 39(24), 1731-1732 (2003).

[13] Boucart, J., Pathak, R., Zhang, D., Beaudoin, M., Kner, P., Sun, D., Stone, R. J., Nabiev, R. F., and Yuen, W., "Long wavelength mems tunable vcsel with inp-inalgaas bottom dbr," IEEE Photonics Technology Letters 15(9), 1186-1188 (2003).

[14] Chang-Hasnain, C. J., "Tunable vcsel," IEEE Journal of Selected Topics in Quantum Electronics 6(6), 978-987 (2000). 